\title{
Quantification of free-living Gyrodactylus salaris in an infested river and consequences for inter-river dispersal
}

\author{
Helga Rachel Høgåsen*, Edgar Brun, Peder A. Jansen
}

National Veterinary Institute, PO Box 750 Sentrum, 0106 Oslo, Norway

\begin{abstract}
Gyrodactylus salaris is a major threat to wild populations of Atlantic salmon Salmo salar in Norway. Inter-river dispersal of this parasite is considered to be mainly linked to infested fish. Dispersal through movement of contaminated water is considered possible but has not been quantitatively evaluated. To approach this problem, we built a simple model to estimate (1) the water concentration of free-living G. salaris and (2) the probability that given water volumes would contain free-living parasites. The concentration was back-estimated from reported experimental observations on the number of salmon juveniles found to be infected after being caged in a heavily infested river in Norway, protected from direct contact with the substrate or fish. Monte Carlo simulation was used to account for uncertainty and variability. The average concentration of G. salaris in the water column during the experiment was estimated to be $0.12 \mathrm{~m}^{-3}(95 \% \mathrm{CI}=0.05$ to 0.24$)$. The probability that a given volume would contain at least 1 parasite was $1.2 \times 10^{-4}\left(95 \% \mathrm{CI}=5 \times 10^{-5}\right.$ to $\left.2.4 \times 10^{-4}\right)$ for $1 \mathrm{l}$, increasing to 0.67 (95\% CI = 0.39 to 0.91 ) for 10000 l. Thus, only high volumes were likely to represent a significant risk. Since we used data from a heavily infested spot, and despite the fact that time and space variations in water concentration of $G$. salaris may occur, we conclude that the risk of inter-river dispersal due to transfer of infested water through common river activities by humans is probably low.
\end{abstract}

KEY WORDS: Gyrodactylus salaris $\cdot$ Risk assessment $\cdot$ River water $\cdot$ Transmission

\section{INTRODUCTION}

Gyrodactylus salaris is considered to be one of the most significant threats to wild populations of East Atlantic stocks of Atlantic salmon Salmo salar (Bakke et al. 2007), and has until 1 January 2008 been recorded from 46 Norwegian rivers (Mo et al. 2008). Norwegian management policy aims to control and eliminate $G$. salaris from infested rivers. The methods that have been applied involve chemical treatment of infested water bodies, either by the use of the biocide rotenone that aims to remove all hosts of G. salaris present in the river and thereby also the parasite, or more recently by the use of aqueous aluminium solutions that aim to eradicate the parasite populations specifically (Soleng et al. 1999b, Poleo et al. 2004). These methods are costly and environmentally controversial and the risk of failing treatments, i.e. not achieving eradication, is high (Bakke et al. 2007). Therefore, preventing further dispersal of G. salaris to non-infested rivers is of great importance.

Dispersal of Gyrodactylus salaris from an infested to a non-infested river necessarily involves (1) the physical movement of parasites between rivers, and (2) the establishment of infestation within the salmon population in the non-infested river. For physical movement a distinction can be made between movement of freeliving parasites and movement of parasites attached to fish hosts or substrate. Whereas dispersal of infested fish is considered to be the main cause of inter-river dispersal of G. salaris in Norway (Johnsen \& Jensen 1991, Jansen et al. 2007), the risk associated with moving water from an infested river to non-infested rivers is unknown. Water may be transported between rivers 
in fishing or canoeing equipment, yachts (e.g. bilge water) and drinking bottles, or when used for washing or watering in the catchment area of another river. In a field experiment, free-living G. salaris in the water column were shown to attach to juvenile salmon (Soleng et al. 1999a). Although this transmission route was found to be less effective than transmission of parasites attached to the substrate or to infested live and/or dead fish (Soleng et al. 1999a), it nevertheless implies that inter-river dispersal of parasites through movement of contaminated water may be possible. However, the whole process of movement of detached G. salaris and subsequent establishment of infestation in a new host population would be restricted by survival time (Olstad et al. 2006). Moreover, it would require sufficient amounts of water containing viable parasites to be moved between rivers within this critical time period.

The proposition that Gyrodactylus salaris disperses between rivers through movement of contaminated water raises important questions with regard to risk management of river activities. For example, the Norwegian Food Safety Authority, which is responsible for managing the G. salaris problem in Norway, is repeatedly confronted with questions regarding disinfestation of sport-fishing gear or the risk involved with boating activities, swimming activities and so on ( $\mathrm{S}$. Johnsen pers. comm.). At present, there are no national regulations aimed at reducing the risk of $G$. salaris dispersal through such activities in Norway, but various regulations are implemented locally. Water may increase the chance of survival of attached parasites by preventing desiccation, but may also contain detached parasites. To approach this last problem, we used experimental data from Soleng et al. (1999a) to estimate and model the concentration of detached $G$. salaris in the water column of a heavily infested river, and to estimate the probability that a given volume of water from this river will contain at least 1 parasite. We used Monte Carlo simulation to account for the uncertainty of the available data as well as the variability inherent to biological systems. The estimates are valid for a heavily infested river, and may therefore be considered a conservative estimate regarding the general risk of moving water from a river infested with $G$. salaris.

\section{MATERIALS AND METHODS}

The concentration of detached Gyrodactylus salaris in water was back-estimated from the proportion of parrs found to be infected after exposure to infested water. This was done by combining 2 different modelling approaches to infestation, the binomial and the Poisson processes, as defined in Vose (2000):

(1) We considered a binomial process in which the known parameters were the number of trials (number of parrs exposed) and the number of successes (number of parrs infested after exposure). The unknown probability of infestation was estimated from these parameters using a Bayesian approach. Since the diagnostic test is not perfect, we corrected the reported number of successes using the sensitivity and specificity of the test.

(2) Once we knew the probability of infestation, we considered a Poisson process in which the known parameters were the exposure volume (volume of water within infective distance of the fish) and the probability that this volume contains at least 1 parasite that could infest the fish (probability of infestation). Considering the attachment efficiency of drifting parasites, the unknown Poisson intensity (concentration of parasites in water) could then be calculated.

(3) We estimated the probability that given water volumes would contain given numbers of parasites by applying a simple Poisson distribution.

To account for uncertainty and variability, a probabilistic model was built in Excel 2002 with @Risk 4.5.2-Professional Edition (Palisade) as an add-in. The choice of input distributions (Table 1) was based on

Table 1. Summary of input values and distributions used in the model

\begin{tabular}{|lcll|}
\hline Variable & Unit & Values & Source of data \\
\hline Diagnostic sensitivity $(S e)$ & & Pert $(0.9,0.95,1)$ & Høgåsen \& Brun (2003) \\
Diagnostic specificity $(S p)$ & 1 & Høgåsen \& Brun (2003) \\
True number of infested fish $\left(N_{\mathrm{i}}\right)$ & & $10+$ negative binomial $(10, \mathrm{Se})$ & Soleng et al. (1999a) \\
$\begin{array}{l}\text { Probability of infestation }\left(P_{\mathrm{i}}\right) \\
\text { Infestive distance }(d)\end{array}$ & $\mathrm{mm}$ & Beta $\left(N_{\mathrm{i}}+1,157-N_{i}+1\right)$ & Soleng et al. (1999a) \\
Efficiency of attachment $(f)$ & $\%$ & 100 in base scenario & Guesstimate by authors \\
& & $0<f \leq 100$ using formula & Assumption by authors \\
Fish length $(L)$ & $\mathrm{cm}$ & Uniform $(6,10)$ & Soleng et al. (1999a) \\
Condition factor $(C F)$ & $\mathrm{g} \mathrm{cm}^{-3}$ & Uniform $(1,1.3)$ & Guesstimate by authors \\
Stream velocity $(u)$ & $\mathrm{cm} \mathrm{s}^{-1}$ & Triangular $(15,20,25)$ & Soleng et al. (1999a) and authors \\
\hline
\end{tabular}


Vose (2000). Latin hypercube sampling was used, and 10000 iterations were run. This number was sufficient to allow convergence of all outputs.

The concentration of Gyrodactylus salaris in the River Glitra, a tributary to the River Lierelva in Buskerud County, was estimated on the basis of an experimental study of G. salaris transmission (Soleng et al. 1999a). In that study, 157 non-infested naïve salmon juveniles were placed individually in small wire mesh cages, hanging above the substrate within larger wire mesh cages (Soleng et al. 1999a). This prevented direct contact between experimental fish and infested fish or substrate. The stream velocity at the surface was reported to be approximately $0.25 \mathrm{~m} \mathrm{~s}^{-1}$, and the water depth approximately $60 \mathrm{~cm}$ (Soleng et al. 1999a). After 24 h, 10 fish were found to be infested, with one G. salaris specimen each (Soleng et al. 1999a).

In accordance with Høgåsen \& Brun (2003), it was assumed that the sensitivity $(\mathrm{Se})$ of the diagnostic method used by Soleng et al. (1999a) was most likely 0.95 , with a minimum value of 0.9 and a maximal value of 1, i.e. 90 to $100 \%$ of the fish infested by Gyrodactylus salaris were detected. This was modeled by a Pert distribution, which is a version of the Beta distribution defined by its minimum, most likely and maximum values. The specificity $(S p)$ of the test was assumed to be $100 \%$, i.e. all fish detected as infested were truly infested by $G$. salaris. The number of truly infested fish that were not detected (false negatives), due to a sensitivity $<100 \%$, was modelled by a negative binomial distribution with parameters 10 (number of infested fish detected) and Se. Therefore, the true number of infested fish $\left(N_{\mathrm{i}}\right)$ was modelled by:

$$
N_{\mathrm{i}} \sim 10+\text { Negative binomial }(10, \mathrm{Se})
$$

where $S e$ was modelled by:

$$
\text { Se } \sim \operatorname{Pert}(0.90,0.95,1)
$$

which corresponds to: Beta $(3,3) \times 0.1+0.9$

The probability of infestation of the experimental fish in Soleng et al. (1999a), $P_{\mathrm{i}}$, was estimated by:

$$
P_{\mathrm{i}} \sim \operatorname{Beta}\left(N_{\mathrm{i}}+1,157-N_{\mathrm{i}}+1\right)
$$

using Bayesian inference with an uninformed prior Beta $(0,1)$.

Free-living Gyrodactylus salaris in the water column were assumed to be randomly distributed with an average concentration, $C$, to be estimated. The variability of $N_{V}$, the number of parasites in a given water volume, $V$, was acknowledged by modelling $N_{V}$ as:

$$
N_{V} \sim \text { Poisson }(C \times V)
$$

Therefore, the probability (p) that $N_{V}$ was higher than zero (which would mean that a water volume $V$ contains at least 1 parasite) was estimated as:

$$
\mathrm{p}\left(N_{V}>0\right)=1-\mathrm{p}\left(N_{V}=0\right)=1-\mathrm{e}^{-C \times V}
$$

As the base scenario, it was assumed that any Gyrodactylus salaris that would drift by an experimental fish at a distance of 0.5 to $1 \mathrm{~mm}$ or less would attach to the fish. This infestive distance $(d)$ corresponds approximately to the body length of 0.7 to $0.8 \mathrm{~mm}$ of G. salaris (T. A. Mo pers. comm.). Our uncertainty about this distance was acknowledged by modelling $d$, as:

$$
d(\mathrm{~mm}) \sim \text { Uniform }(0.5,1)
$$

The volume of water flowing within the infestive distance $d$ over $24 \mathrm{~h}$, was denoted the infestive volume, $V_{\mathrm{i}}$. The probability that a fish became infested could then be equalised to the probability that $V_{\mathrm{i}}$ contained at least 1 parasite:

$$
P_{\mathrm{i}}=\mathrm{p}\left(N_{V_{\mathrm{i}}}>0\right)=1-\mathrm{e}^{-C \times V_{\mathrm{i}}}
$$

From which the concentration of parasites, $C$, could be calculated:

$$
C=-\left(1 / V_{\mathrm{i}}\right) \times \ln \left(1-P_{\mathrm{i}}\right)
$$

The efficiency of attachment of detached parasites drifting within the infestive distance, $f$, was assumed to be $100 \%$. If another attachment efficiency is considered more appropriate, the estimated concentration may be corrected accordingly:

$$
C_{f}=C_{0} / f
$$

where $C_{f}$ is the concentration of parasites, $C_{0}$ is the estimated concentration of parasites assuming a $100 \%$ efficiency of attachment, and $f$ is the actual attachment efficiency. For example, if only half of the free-living parasites would actually attach $(f=0.5)$, the concentration of parasites in the water would be twice as high as estimated by the base model.

To calculate the infestive volume over $24 \mathrm{~h}, V_{\mathrm{i}}$, we used data on water velocity, fish length and surface area. According to Wells \& Pinder (1996), the surface area $\left(S\right.$ in $\mathrm{mm}^{2}$ ) of Atlantic salmon parr of mass ( $M$ in $\mathrm{g}$ ) in the range 0.19 to $11.2 \mathrm{~g}$ is given by:

$$
S=1582 \times M^{0.54}
$$

Soleng et al. (1999a) report that the experimental fishes' length $(L$ in $\mathrm{cm}$ ) was in the range 6 to $10 \mathrm{~cm}$. Therefore we used the variability distribution:

$$
L \sim \text { Uniform }(6,10)
$$

The condition factor $\left(C F=M(\mathrm{~g}) \times 100 / L(\mathrm{~cm})^{3}\right)$ was assumed to be in the range 1 to 1.3 since the experimental fish were hatchery-reared Atlantic salmon parr age $1+. M(g)$ was calculated from the formula:

$$
M=C F \times L^{3} / 100, \text { with } C F \sim \text { Uniform }(1,1.3)
$$


The instant infestive volume was obtained by multiplying $S$ by $d(\mathrm{~mm}) . V_{\mathrm{i}}$ was then obtained by multiplying the instant infestive volume by the number of times this volume is renewed in $24 \mathrm{~h}$, based on a fish length $L(\mathrm{~cm})$ and a stream velocity $u\left(\mathrm{~cm} \mathrm{~s}^{-1}\right)$ :

$$
V_{\mathrm{i}}\left(\mathrm{mm}^{3}\right)=(S \times d)(3600 \times 24 u / L)
$$

The uncertainty about the stream velocity at the level of the fish, $u$, was described by a triangular distribution parameterised on the basis of the reported stream surface velocity of $25 \mathrm{~cm} \mathrm{~s}^{-1}$ :

$$
u\left(\mathrm{~cm} \mathrm{~s}^{-1}\right) \sim \text { Triangular }(15,20,25)
$$

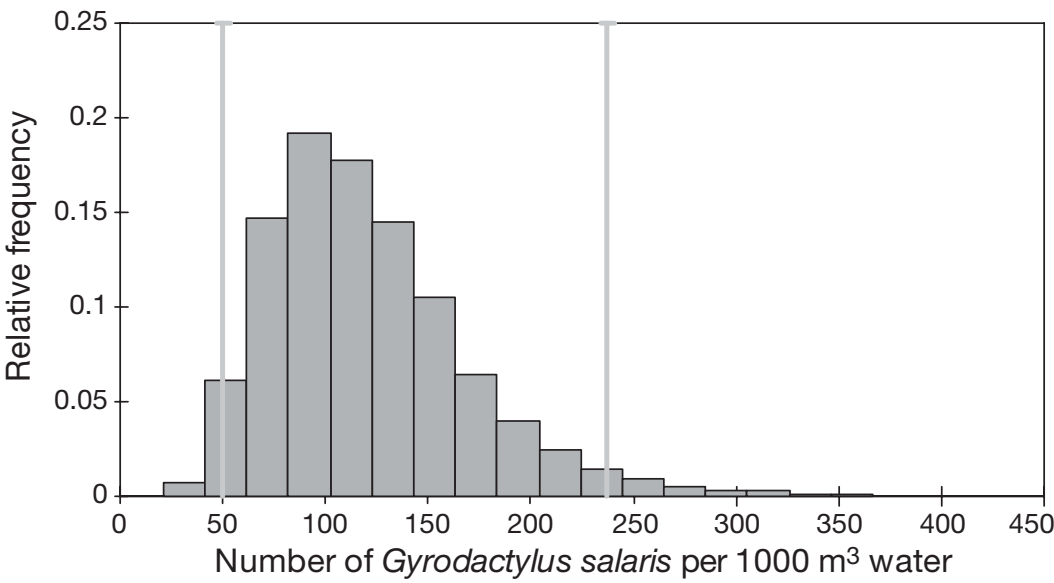

Fig. 1. Gyrodactylus salaris. Uncertainty distribution of the number of parasites per $1000 \mathrm{~m}^{3}$ of River Glitra water during the field experiment by Soleng et al. (1999a). Vertical grey lines indicate the $95 \%$ CI

Once the concentration $C$ was estimated, the probability that a given volume $(V)$ of water from the river contains at least 1 parasite was calculated based on the Poisson distribution formula:

$$
\mathrm{p}\left(N_{V}>0\right)=1-\mathrm{p}\left(N_{V}=0\right)=1-\mathrm{e}^{-C \times V}
$$

which has the following property:

$$
1-\mathrm{e}^{-C \times V} \approx C \times V \text {, when } C \times V \text { is small }
$$

Sensitivity analysis based on the Spearman rank correlation coefficient $(\rho)$ calculations was used to analyse how variations in the stochastic variables influenced the result.

\section{RESULTS}

\section{Concentration of Gyrodactylus salaris in the water column}

The average concentration of Gyrodactylus salaris in the water column during the experiment was estimated to be $0.12 \mathrm{~m}^{-3}$. Expressed as number per $1000 \mathrm{~m}^{3}$, the $95 \%$ CI was 50 to 236 , the range was 22 to 450 , and the median value was 112 . The full uncertainty distribution is illustrated in Fig. 1.

\section{Probability that water taken from the river contains detached Gyrodactylus salaris}

Mean probabilities estimated for selected volumes and abundances of parasites are shown in Table 2. Volumes up to $100 \mathrm{l}$ were unlikely to contain any parasites ( $\mathrm{p} \leq$ 0.012), whereas high volumes were likely to contain 1 or a few parasites. For example, 100001 were expected to contain no parasites in $33 \%$ of cases, 1 parasite in $34 \%$ of cases, and 2 to 5 parasites in $33 \%$ of cases.

Table 2. Gyrodactylus salaris. Probability distribution of the number of parasites expected in given water volumes of River Glitra during the field experiment by Soleng et al. (1999a)

\begin{tabular}{|lcccc|}
\hline \multirow{2}{*}{$\begin{array}{l}\text { No. of } \\
\text { parasites }\end{array}$} & 1 & \multicolumn{5}{c|}{ Water volume $(1)$} \\
\cline { 2 - 5 } & \multicolumn{5}{c}{100} & 1000 & 10000 \\
\hline 0 & 0.9999 & 0.99 & 0.89 & 0.33 \\
1 & $1.2 \times 10^{-4}$ & $1.2 \times 10^{-2}$ & 0.01 & 0.34 \\
2 & $8.4 \times 10^{-9}$ & $8.3 \times 10^{-5}$ & $7.2 \times 10^{-3}$ & 0.2 \\
3 & $4.6 \times 10^{-13}$ & $4.5 \times 10^{-7}$ & $3.8 \times 10^{-4}$ & $8.7 \times 10^{-2}$ \\
4 & $2.1 \times 10^{-17}$ & $2.1 \times 10^{-9}$ & $1.7 \times 10^{-5}$ & $3.2 \times 10^{-2}$ \\
5 & $8.9 \times 10^{-22}$ & $8.7 \times 10^{-12}$ & $7.0 \times 10^{-7}$ & $1.1 \times 10^{-2}$ \\
$>5$ & $3.5 \times 10^{-26}$ & $3.4 \times 10^{-14}$ & $2.8 \times 10^{-8}$ & $4.8 \times 10^{-3}$ \\
$>0$ & $1.2 \times 10^{-4}$ & $1.2 \times 10^{-2}$ & 0.10 & 0.67 \\
\hline \multicolumn{5}{c}{} \\
\hline
\end{tabular}

The mean probability that a given volume of water $V$ contains at least 1 detached Gyrodactylus salaris may also be calculated as:

$$
\mathrm{p}\left(N_{V}>0\right)=1-\mathrm{e}^{-0.12 \times V} \approx 0.12 \times V\left(\mathrm{~m}^{3}\right)
$$

The linear approximation is valid for volumes up to 100 l. Ordinary leisure activities like fishing, drinking and canoeing may be associated with the transfer of small water volumes between rivers, typically $<1 \mathrm{l}$ per 'event'. The probability that $1 \mathrm{l}$ contains at least $1 \mathrm{G}$. salaris was estimated to be $1.2 \times 10^{-4}\left(95 \% \mathrm{CI}=5 \times 10^{-5}\right.$ to $2.4 \times 10^{-4}$ ). However, if these activities are frequent, the total amount of water transferred may become significant.

\section{Sensitivity analysis}

The probability that parasites were present in a given volume was positively correlated with the probability of infection $P_{\mathrm{i}}(\rho=0.76)$ and negatively corre- 
lated with the infective distance $d(\rho=-0.51)$, the stream velocity $u(\rho=-0.26)$ and fish length $L(\rho=$ $-0.23)$. Its correlation with condition factor, true number of infected fish and test sensitivity was low $(|\rho|<$ 0.2), indicating that any uncertainties about these parameters have little effect on the output.

\section{DISCUSSION}

The concentration of free-living Gyrodactylus salaris drifting in the water column of an infested river was estimated in order to assess the risk of inter-river dispersal through movement of infested water in association with common river activities such as sport fishing, boating activities, swimming activities and so on. Results are based on an experiment performed in the river Glitra during summer (Soleng et al. 1999a), when the level of infestation may be considered high compared to average infested rivers, and represent therefore a conservative estimate. In this regard, the present results indicate that the concentration of free-living G. salaris in an infested river is generally low, and therefore the probability of spread through common river activities is low. With the given model assumptions, a concentration of approximately 1 detached $G$. salaris per $8 \mathrm{~m}^{3}$ (range $=2$ to $46 \mathrm{~m}^{3}$ ) was estimated from the experiments conducted in the River Glitra (Soleng et al. 1999a). It was found that 11 of water would contain at least 1 G. salaris in approximately 1 out of 8000 trials.

These results are based on a number of assumptions necessary to simplify the complexity of biological systems for modelling purposes. When conducting riskassessment studies, there is generally limited data available, and often available data are not directly applicable since they are gathered for purposes other than risk assessment. To overcome these limitations, we used Monte Carlo simulation techniques to account for the uncertainty related to a number of parameters in the model, as well as the variability inherent to biological systems. Moreover, sensitivity analysis was conducted to assess which of the model inputs had a strong effect on the model output, thereby assessing the impact of the different uncertainties on the results of the model. This information can be used to direct resources into future research.

The estimated probability of infestation of fish subjected to the experimental conditions in Soleng et al. (1999a) was shown to be strongly positively correlated with the estimated probability that given volumes of river water would contain parasites. This estimate is based on relatively limited experimental data gathered from only one location. Hence, we acknowledge that the actual estimate is valid only for the given river at the given time period. However, the location where the experiments in Soleng et al. (1999a) were conducted is known to have a relatively dense population of Atlantic salmon juveniles with high Gyrodactylus salaris prevalence and intensity, particularly during summer (Jansen \& Bakke 1993). Still, even under these circumstances, the estimated concentration of freeliving parasites was so low that we are confident that the result is highly relevant to assessments of the risk of spreading G. salaris by movement of small volumes of water from most infested rivers. Alternatively, a more specific estimate can be obtained by conducting a similar experiment as Soleng et al. (1999a) in the river of interest and applying the model described in the present paper. In this case the model could be simplified by using the actual fish weight distribution directly, rather than deriving it from fish length and estimated condition factor. Increased precision may be obtained by increasing the number of fish exposed to the river flow in the field experiment.

The infestive distance, the maximum distance to the fish from which a free-living parasite may attach to the fish, was also shown to have a significant impact on the model outputs. The correlation was negative, which means that when the distance was lower, the water concentration of parasites and the probability that given water volumes contained parasites was higher, since less water was running at appropriate distance during the experiment. Based on the body length of Gyrodactylus salaris ( 0.7 to $0.8 \mathrm{~mm}$ ), we assumed that the infestive distance was in the range 0.5 to $1 \mathrm{~mm}$. According to Olstad et al. (2006), G. salaris is capable of extending its body length to at least 4 times its normal length while in a search mode, a behaviour which Olstad et al. (2006) coupled with transmission. This may indicate that the present model underestimates the transmission distance of free-living parasites in the field experiment, which, in turn, will overestimate the concentration of parasites in the water.

Stream velocity and fish length had a moderate effect on the model output, which means that although exact water velocity at the level of the fish, and exact fish length, are uncertain, these are not likely to critically affect conclusions. Variations in the last 3 inputs, condition factor, the true number of infested fish and diagnostic sensitivity, had little effect on the output in the present model.

The present estimates were obtained assuming an attachment efficiency of $100 \%$ for parasites drifting by closer than 0.5 to $1 \mathrm{~mm}$ from the experimental fish. Although it underestimates the number of drifting parasites, this simplification was considered useful and adequate because of the risk focus of our approach and the complexity of the phenomenon. Attachment efficiency of Gyrodactylus salaris is known to be $<100 \%$. Olstad et al. (2006) found that $50 \%$ of 
attempted transfers of $G$. salaris from live fish were successful in infesting new naïve hosts, whereas only 1 of 14 parasites previously kept for $24 \mathrm{~h}$ in vitro was able to re-infect a new host. From a risk assessment point of view, it is pertinent to focus on the number of parasites that actually would attach to a host when drifting close enough, rather than including non-infestive parasites. In this view, the present approach can be seen as a biological test, rather than a mere count of parasites of unknown biological significance. When released into a new river, the attachment efficiency of drifting parasites would at most be that observed in the source river, since it decreases with time as the freeliving parasites are starving (Olstad et al. 2006), and the present estimates therefore remain conservative. Olstad et al. (2006) found a maximum survival time for detached G. salaris of $2.5 \mathrm{~d}$ in cold water $\left(3^{\circ} \mathrm{C}\right)$ and $1 \mathrm{~d}$ in warmer water $\left(18^{\circ} \mathrm{C}\right)$. The population half-life was only $28 \mathrm{~h}$ at the coldest temperature $\left(3^{\circ} \mathrm{C}\right)$. On the other hand, they would probably attach to the substrate, from which they may attach more efficiently to new hosts. If of interest, the total number of drifting parasites present, infestive or not, could be calculated from the linear relationship between attachment efficiency and the concentration of free-living parasites, as estimated from the present model. If $50 \%$ of the parasites that drifted by the experimental fish within the infestive distance actually attached, then the concentration in the river water was actually twice as high as the present estimates. Accordingly, the probability that a given volume of water (e.g. 25 l) contains at least 1 parasite is the same as estimated for twice the volume (50 1), given $50 \%$ attachment efficiency in the experiment.

Inter-river dispersal of Gyrodactylus salaris through movement of free-living parasites in water requires a full series of consecutive events: (1) the water moved from an infested to a non-infested river contains $G$. salaris; (2) the water ends up in the non-infested river within a restricted time period; (3) the non-infested river is inhabited by susceptible hosts; (4) at least 1 moved parasite comes into contact, attaches to and reproduces on a host; and (5) parasites spread to new hosts within the new river. There is no indication that G. salaris has ever dispersed between rivers due to such a series of events in Norway. However, there has been a lot of public attention paid to the possibility of G. salaris dispersal through movement of water in connection with common river activities such as angling, boating activities, swimming activities and so on. The present study suggests that unless large volumes of water are transferred between rivers, the probability of transferring G. salaris through infested water is low. Ordinary leisure activities like fishing, drinking and canoeing are associated with the transfer of only small water volumes between rivers, typically $<1$ l per 'event'. They may add up to large total amounts of water being transferred only if the activities are very frequent or happen over a long period of time. Even in that case, the next steps necessary for inter-river dispersal would limit the risk. Therefore, we conclude that the risk of inter-river dispersal due to transfer of infested water through common river activities is probably low. The low water concentration of parasites also indicates that the probability of attachment of drifting parasites to immersed equipment or swimmers is low. In comparison, the risk of inter-river transmission of $G$. salaris by migrating Atlantic salmon smolts or other species could be significant (Høgåsen \& Brun 2003, Peeler et al. 2004). All results support the hypothesis by Jansen et al. (2007) that G. salaris dispersal in Norway is due to either anthropogenic movement of infested salmon, or migration of infested salmon in fjord systems.

If specific estimates of the concentration of free parasites in water are needed, the method described in the present paper can be used to provide a first approximation after performing a relatively simple field experiment similar to that in Soleng et al. (1999a). The present approach should preferentially be validated by some direct quantitative measurement in water, although the low concentration of parasites makes such an approach technically challenging.

\section{LITERATURE CITED}

Bakke TA, Cable J, Harris PD (2007) The biology of gyrodactylid monogeneans: the 'Russian-doll killers'. Adv Parasitol 64:161-378

Høgåsen HR, Brun E (2003) Risk of inter-river transmission of Gyrodactylus salaris by migrating Atlantic salmon smolts, estimated by Monte Carlo simulation. Dis Aquat Org 57:247-254

> Jansen PA, Bakke TA (1993) Regulatory processes in the monogenean Gyrodactylus salaris Malmberg - Atlantic salmon (Salmo salar L.) association. 1. Field studies in southeast Norway. Fish Res 17:87-101

Jansen PA, Matthews L, Toft N (2007) Geographic risk factors for inter-river dispersal of Gyrodactylus salaris in fjord systems in Norway. Dis Aquat Org 74:139-149

Johnsen BO, Jensen AJ (1991) The Gyrodactylus story in Norway. Aquaculture 98:289-302

Mo TA, Norheim K, Jansen PA (2008) The surveillance and control programme for Gyrodactylus salaris in Atlantic salmon and rainbow trout in Norway. In: Brun E, Hellberg $\mathrm{H}$, Sviland S, Jordsmyr HM (eds) Surveillance and control programmes for terrestrial and aquatic animals in Norway. Annual report 2007. National Veterinary Institute, Oslo, p 145-148

> Olstad K, Cable J, Robertsen G, Bakke TA (2006) Unpredicted transmission strategy of Gyrodactylus salaris (Monogenea: Gyrodactylidae): survival and infectivity of parasites on dead hosts. Parasitology 133:33-41

Peeler EJ, Gardiner R, Thrush MA (2004) Qualitative risk assessment of routes of transmission of the exotic fish par- 
asite Gyrodactylus salaris between river catchments in England and Wales. Prev Vet Med 64:175-189

Poleo ABS, Schjolden J, Hansen H, Bakke TA, Mo TA, Rosseland BO, Lydersen E (2004) The effect of various metals on Gyrodactylus salaris (Platyhelminthes, Monogenea) infections in Atlantic salmon (Salmo salar). Parasitology 128:169-177

Soleng A, Jansen PA, Bakke TA (1999a) Transmission of the monogenean Gyrodactylus salaris. Folia Parasitol (Praha) 46:179-184

Editorial responsibility: Julie Bebak,

Auburn, Alabama, USA
Soleng A, Poleo ABS, Alstad NEW, Bakke TA (1999b) Aqueous aluminium eliminates Gyrodactylus salaris (Platyhelminthes, Monogenea) infections in Atlantic salmon. Parasitology 119:19-25

Vose D (2000) Risk analysis. A quantitative guide. John Wiley \& Sons, Chichester

Wells PR, Pinder AW (1996) The respiratory development of Atlantic salmon. 1. Morphometry of gills, yolk sac and body surface. J Exp Biol 199:2725-2736

Submitted: May 22, 2008; Accepted: June 25, 2009

Proofs received from author(s): November 10, 2009 